\title{
Lamb Modes Detection Using Cumulative Shannon Entropy with Improved Estimation of Arrival Time
}

\author{
Francisco Sagasta $^{1} \cdot$ Kong Fah Tee $^{1}\left[\right.$ [Dosa Piotrkowski ${ }^{2,3}$
}

Received: 15 November 2017 / Accepted: 1 February 2019 / Published online: 15 February 2019

(c) The Author(s) 2019

\begin{abstract}
Non-destructive testing techniques for damage localization constitute a key aspect of structural health monitoring (SHM) systems. The acoustic emission (AE) method can be used for SHM and its location capability is considered as one of the most powerful qualities. This paper presents a novel method for distinguishing between AE transient and noise signals and this proposes a very promising procedure to detect the Lamb modes of AE signals, which provides an improvement in the localization of AE sources. The AE signals were generated on an aluminium flat bar and a plate using the normalised Hsu-Nielsen source. For a successful localisation of the AE sources, both the noise signal discrimination and detection of the beginning of the signal are crucial. The source locations were determined using the conventional Time of Arrival technique and a novel approach based on Shannon entropy of each AE signal. A sharp and substantial change in the cumulative Shannon entropy (CSE) at the instant of arrival of the A0 and S0 Lamb modes was observed. In addition, a comparison of the results using the first Threshold crossing and CSE of the AE signals illustrated that the new proposed method detects more accurately the location of AE sources and reduces the ambiguity introduced by arrival times of noisy signals in both studied specimens.
\end{abstract}

Keywords Acoustic emission · Source location · Time of arrival · Entropy $\cdot$ Lamb waves $\cdot$ Noise signals

\section{Introduction}

Structural Health Monitoring (SHM) [1] and non-destructive testing (NDT) [2] play very important roles in studying degradation in materials and building structures. Material and structural properties degrade over time due to fatigue processes under prolonged load conditions and the influence of environmental circumstances such as extreme temperature values and the action of chemical agents. Thus, the evaluation of structural health during maintenance of materials, machines and structures systems must be performed [3,4]. The majority of maintenance and inspection activities for early damage detection are principally based on NDT methods. Acoustic emission (AE) is one of the few

Kong Fah Tee

K.F.Tee@gre.ac.uk

1 Department of Engineering Science, University of Greenwich, Kent, UK

2 Departmento de Matemática, Facultad de Ingeniería, Universidad de Buenos Aires, Buenos Aires, Argentina

3 Escuela de Ciencia y Tecnología, Universidad Nacional de San Martín, Buenos Aires, Argentina well established NDT methods that can be implemented for real-time damage monitoring and other SHM applications because it is able to provide information on damage progression [5-12].

Unlike AE, where inspection is localized, in the guided wave system (or long range ultrasound) the inspection is done using a ring of transducers to emit low frequency ultrasonic waves that travel large distances from a single point of application. It is mainly used as a non-conventional type of NDT used for the detection of material losses in pipes. There also are important contributions that deal in damage detection and location using combination of $\mathrm{AE}$ and guided waves signals in large structures. Flynn et al. [13] formulate a maximum-likelihood estimate of damage location for guided-wave structural health monitoring using a minimally informed Rayleigh-based statistical model of scattered wave measurements. Courtier et al. [14] present a practical modeling approach that aids the understanding of how wave propagation within a structure affects the performance of an acoustic emission system.

Our work, while not addressing the complex issue of damage detection in large structures, does face a crucial aspect in the detection of damage, which is the location of 
AE sources; one of the fundamental challenges in AE and SHM. The information of arrival time of AE signals is very important in terms of analysis of the source, identification and localization of the origin mechanism $[15,16]$. It can be described as the time when the first significant energy of a particular phase is registered by a sensor or a time point where the difference from noise occurs first [17]. Previous researchers have stated in the last few decades that this is laborious because it needs a major effort due to the high complexity and variability of AE waveforms. Therefore, various signal processing methods have been developed for AE source localization e.g. triangle technique [9], cluster analysis [18], delta $T$ mapping which determines an area of interest and constructs a grid system to calculate a $\Delta t$ map based on the collected arrival time data from artificial sources for each grid node $[15,19,20]$, probabilistic methods [21], neural network [22], etc. Autoregressive methods have also been developed, for example, the Akaike information criteria (AIC) which can be divided into two locally stationary processes (noise-signal) in signal modelling [23, 24]. The Akaike information criterion (AIC) is of great importance in seismology to detect and pick up the P-wave arrival, however it requires an appropriate time window, otherwise it will detect the wrong P-wave arrival. There are other techniques that work in the frequency domain such as the cross-correlation technique [25], which identifies energy arrival across frequency range, and the wavelet transform theory that works in the time-frequency plane. These methods, however, do not provide very high accuracy in the determination of arrival times of the different modes in complex structures due to the existence of reflections [26]. A hybrid technique has been proposed [27] where the sources can be located in anisotropic plates in two steps. The propagation of the wave in a straight line is assumed in the first step to find the initial source and the second step is to optimize the location accuracy made previously in the first step. All of the methods have advantages and limitations.

All researchers agree that robust AE localization algorithms become challenging when AE signals are significantly affected by multiple wave propagation effects such as reflections, attenuation, mode conversions or dispersion. The main causes of error in the localization and the associated uncertainties are clearly outlined in [19, 20]: (i) deterioration of the waves on their journey from the source to the sensor, resulting in further difficulties in determining the time of arrival (TOA); (ii) the speed of propagation: if the medium is heterogeneous, there will not be a unique propagation velocity and if the medium is dispersive (Lamb waves, for example), the speed is not unique but depends on the frequency; (iii) conversion of propagation modes: sometimes, there are conversion modes or modes can arrive overlapping; (iv) the size of the sensor: its influence depends on the wavelength of the propagation wave and the angle of incidence. Moreover, the frequency content of the received waves is also distorted due to the sensor size, increasing the possible distortion due to scattering or reflections. These phenomena are well described in [28].

It is well known that $\mathrm{AE}$ waves propagate through a structure in different modes. The entire thickness of the specimen will be crucial and decisive for Lamb modes appearing. The separation of these modes during data acquisition and/or posterior data analysis enables one to extract information about the signal classification, damage mechanism, identification of source mechanisms, source location, etc. The plate theory establishes that $\mathrm{AE}$ waves propagate through plates in two modes, the extensional and the flexural modes, also respectively called symmetric $(\mathrm{Si})$ and anti-symmetric (Ai) modes.

The present work is devoted to detect the Lamb modes of $\mathrm{AE}$ signals in $\mathrm{Al}$ samples. Only the fundamental modes $\mathrm{S} 0$ and $\mathrm{A} 0$ are considered according to the dispersion curves in Aluminium plates, verified with the Vallen Systeme Dispersion software R2011 [29]; the frequency band of the employed sensor (medium frequency $150 \mathrm{kHz}$ ) was consequently selected.

The S0 and A0 modes have been clearly recognized in AE signals coming from pencil lead breaking performed in different tests $[30,31]$. These modes travel at different velocities and exhibit different dispersion characteristics [16]. Estimation of arrival times from superposed different modes or superposed different frequency components that correspond to different velocities, will inevitably lead to source localization errors. The objectives of this study are: firstly, to distinguish between $\mathrm{AE}$ transient and noise signals which is a situation needed in any AE test.; secondly, to analyse signals in order to evaluate and identify the true TOA for the modes in Lamb waves. To achieve both objectives a cumulative entropy method, named here Cumulative Shannon Entropy (CSE) is employed.

Entropy is basically a measure of disorder in a physical system. It is well known that Shannon entropy is the extended application for Boltzmann-Gibbs (B-G) entropy in information theory. As an important index in thermodynamics, B-G entropy represents the measurement for the uncertainty of an N-energy-level system [32,33]. Shannon was inspired to induce B-G entropy to measure the information loss in information theory [32, 34-37]. Entropy has been successfully applied by different authors to AE signal processing [29, 38-40].

Noise signals (white noise would be an extreme case) come from a greater number and variety of sources, then it is expected that the present approach of analysing entropy allows one to distinguish between $\mathrm{AE}$ authentic transient and noise signal. After identifying the true signals of damage, one can proceed studying the arrival of the wave modes from AE waveform. 
In this paper, the entropy concepts are applied to discriminate noise signals and to detect, classify and identify the $\mathrm{S} 0$ and $\mathrm{A} 0$ modes of the AE signals; the evolution of entropy along each true AE signal is defined. The non-normalized entropy definition, is applied for convenience, as is later explained in Sect. 3, as in previous work concerning dynamic, complex and nonlinear systems [41, 42].

Finally, TOA detected with the CSE method and standard First Threshold Crossing (FTC) technique are compared. In spite of the fact that more sophisticated TOA methods are in the literature, listed in our references, our interest is to present an efficient method, based on physical grounds, and suitable for being introduced in an automated code in future work, and compare its results with a well-established code based on FTC. This research was carried out on two specimens: thin aluminium flat bar and plate. AE events were generated by normalised artificial sources. Results of comparison are promising.

\section{Source Localization Technique}

One of the major advantages of the AE method is that, by analysing data from suitable transducers, one can locate the source of the observed instability. AE signals received by various transducers are processed to provide parameters such as arrival time sequence, signal amplitude, etc. To achieve precise spatial location of the AE sources, a suitable algorithm must be developed. Locating methods imply mathematical procedures starting with physical observation of the AE event, which is then expressed in terms of parameters of the hypocentre. One of the most used algorithms in locating AE signals is the time of arrival (TOA) [9, 43-45]. This type of algorithm uses exclusively the information of arrival time, which may correspond to any type of wave, but usually the $\mathrm{P}$ wave is used in bulk waves normally called the longitudinal wave in NDT and the S0 mode in Lamb waves because they are the fastest propagating waves.

The source localization method can employ several transducers. The number of them will depend on the type of localization required: linear or one dimension, planar or two dimensions, or 3D localization. In all cases the minimum required number of sensors is obtained as the dimension number plus one, i.e. no $_{\text {Sensors }}=$ no $_{\text {Dimensions }}+1$. In the case that the number of sensors is higher than the minimum, an over-determined system with various solutions will be obtained and it can be solved with other procedures, for example least squares technique. The use of a higher number of sensors provides more accurate and reliable results but the computation time increases. In a localization process, the following steps can be distinguished (Fig. 1) before providing the final result (the location of the source, at one point or small area).

\subsection{Determining the Time of Arrival}

Determining the TOA is a fundamental aspect in the whole localization process because it constitutes a major source of errors. It is still a topic of interest in current research; many of the contributions are of great and increasing conceptual or experimental complexity [20,46,47]. Out interest is to compare our results with the most commonly used method in commercial testing equipment for $\mathrm{AE}$ technique is to set the time of arrival as the first threshold crossing (FTC), that is, TOA $=$ FTC $[9,43-45]$. However, in this case the threshold is decided based on experience. As can be seen in Fig. 2, where th is the threshold. If the threshold is very high, the arrival time is overestimated, and if it is too low, it may be determined incorrectly due to background noise. In Fig. 2, $0 \mathrm{~dB}$ would mean the peak of the complete event.

Besides, other systematic errors produced by the sampling frequency used in the data acquisition system exist. This is a systematic uncertainty that generates an error lower than $d t\left(1 / f_{s}\right)$, where $d t$ is the sampling error and $f_{s}$ is the sampling frequency [21, 48, 49]. It should be noted that, in this paper the systematic error due to the digital time resolution is considered and corrected. Figure 3 shows the systematic sampling frequency error for a discretized signal when FTC is considering as TOA.

\subsection{Event Builder}

AE activity is recorded by the acquisition equipment as sequences of individual hits. These sequences, however, do not provide information from which source they came and therefore must be properly grouped in events, assuming that each event is a set of hits that come from the same source of AE. That is to say, at several times a hit is detected, since the threshold is surpassed. Consecutive hits occurring within an interval $\Delta \tau$ are supposed to constitute one separate $\mathrm{AE}$ event, originated in the same source. The interval $\Delta \tau$ is estimated as proportional to the ratio between the maximum source-sensor distance and the medium velocity of $\mathrm{AE}$ waves in the sample. The constant of proportionality was
Fig. 1 Steps in a localization process

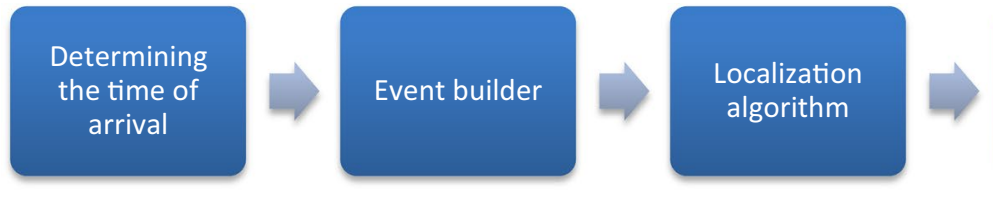

Result: Position

or area where

the source is

located 
Fig. 2 Determining the time of arrival using TOA $=$ FTC for different thresholds

Fig. 3 Systematic sampling error due to discretization
Whole AE signal

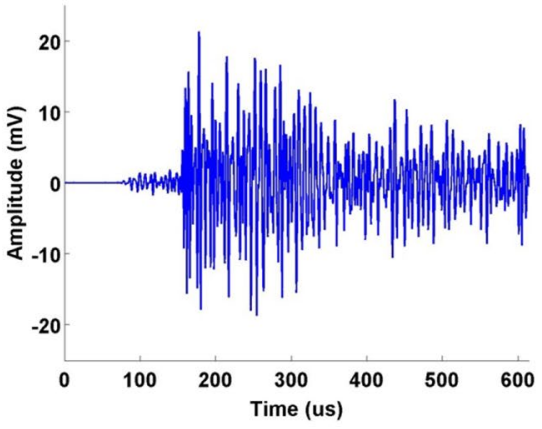

$\mathrm{TH}=25 \mathrm{~dB}(70 \mu \mathrm{s})$

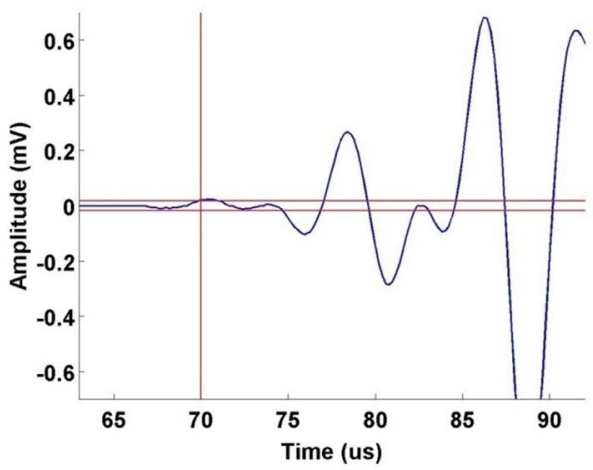

$\mathrm{TH}=45 \mathrm{~dB}(\mathbf{7 7 . 8} \mu \mathrm{s})$

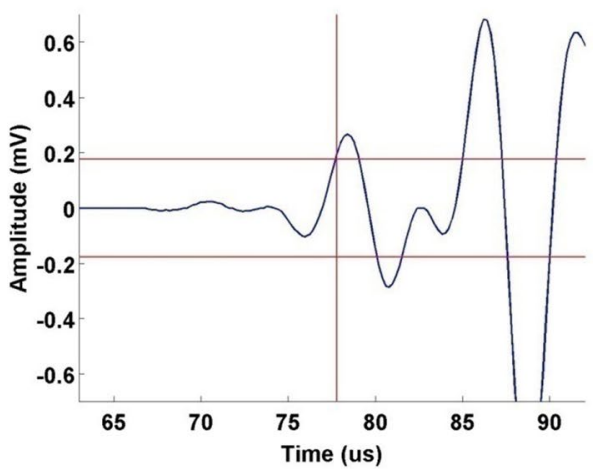

Zoom AE signal (true arrival time $=66.8 \mu \mathrm{s}$ )

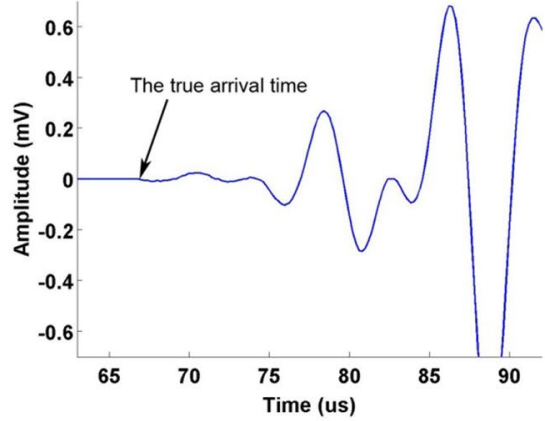

$\mathrm{TH}=35 \mathrm{~dB}(75.2 \mu \mathrm{s})$

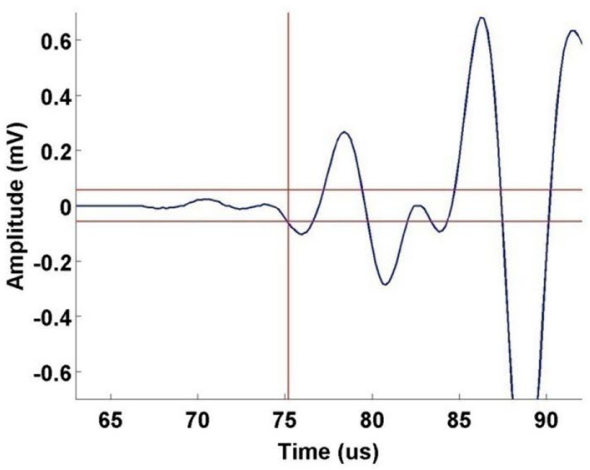

$\mathrm{TH}=50 \mathrm{~dB}(85.4 \mu \mathrm{s})$

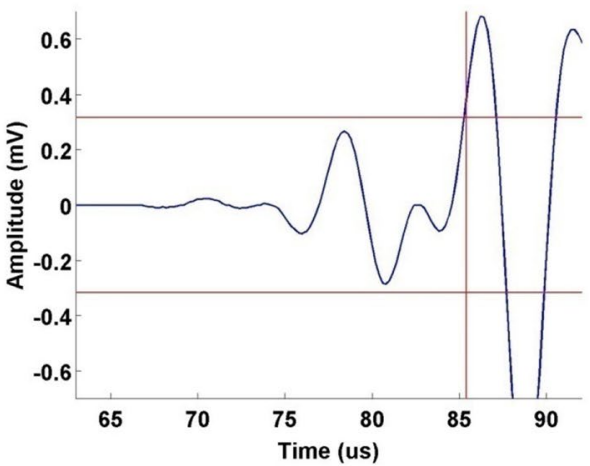

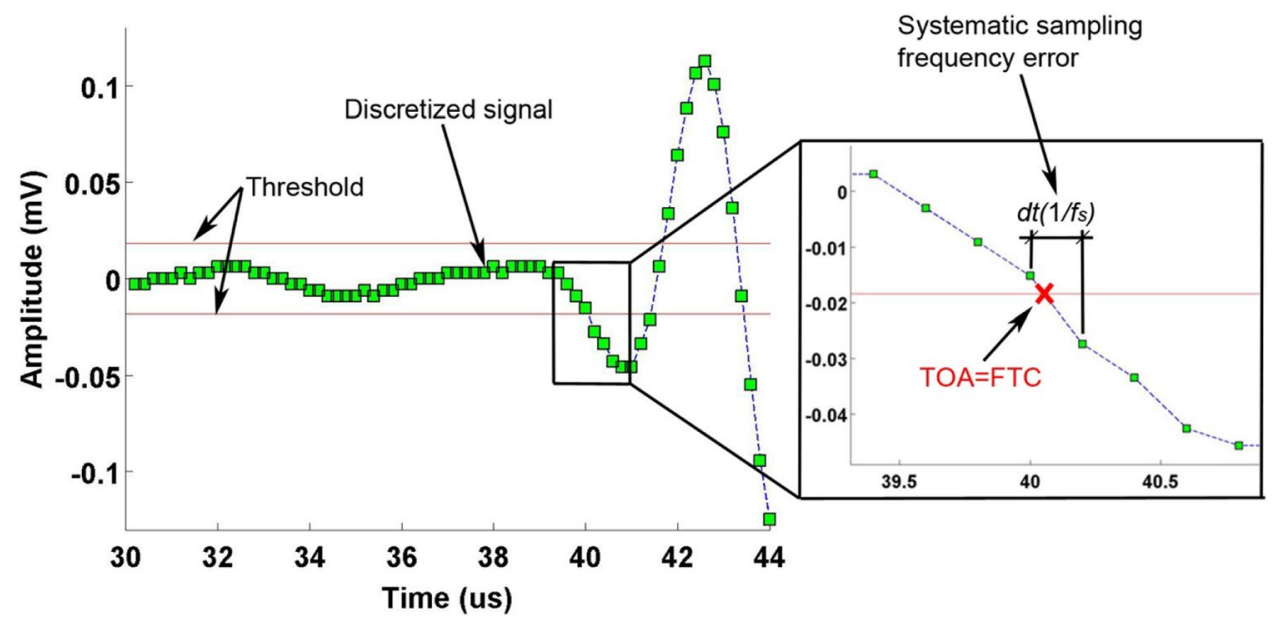


taken as 1.1. The clustering of hits is key to the subsequent localization process. This grouping should be done with the Event Definition Time and parameters are introduced by the user $[44,50]$. An event consists of a first hit and subsequent hits or slaves, which are formed according to the described criteria.

\subsection{Localization Algorithm}

There are many specific localization algorithms for particular types of geometric structures (linear, planar, etc.). The algorithm basically determines the location. To perform this requires: (i) Sensors location (their coordinates); (ii) The set of events, along with the arrival times for each hit of the event. Usually the difference in arrival time is used between a particular hit and the first hit of the event; (iii) The propagation speed of waves; (iv) The minimum number of hits that the event must have to be located. All algorithms require a minimum number of sensors.

Once the arrival times for different hits of each event are known, a specific localization algorithm is required to determine the precise position of the source (if it is a point algorithm) or the area of the source (if it is a zonal algorithm). The travel time difference method is the most frequently used. $\Delta t$ is defined as the time interval between the detected arrival of an AE wave with two sensors $[9,15,43$, 50]. Assuming the validity of the linear localization algorithm and using the minimum number of transducers, an $\mathrm{AE}$ event occurs somewhere in the material and after that the resulting stress waves propagate in both directions at the same constant velocity, which is given in Eq. 1:

$d=\frac{1}{2}(D-\Delta t V)$

where $D$ is the distance between transducers, $V$ is the constant wave velocity, $\Delta t$ is the difference of arrival time between the first hit of the event and the second one and $d$ is the coordinate of the wave located from the first sensor. The linear case is the most appropriate when the transducer separation is large compared to the diameter of the test object [9].

Considering several transducers mounted on an infinite plane and assuming the ideal condition where the stress waves propagating from an AE source travel at constant velocity in all directions, the location of sources in two dimensions can be expressed by their polar coordinates $(R, \theta)[6,9]$ as in Eq. 2:

$R=\frac{1}{2} \frac{D^{2}-\Delta t^{2} V^{2}}{\Delta t V+\cos \theta}$

Equation 2 corresponds to a hyperbola passing through the source location $\left(X_{S}, Y_{S}\right)$. Any point of the hyperbola satisfies the input data. This input data includes an event of a minimum of three hits and two times difference measurements (between the first and second hit at $\mathrm{AE}$ sensors and the first and third hit at AE sensors). A sketch of location in an infinite plane can be seen in Fig. 4. In the next section the method applied in the present paper is described.

\section{AE Signal Processing: Cumulative Shannon Entropy}

\subsection{Shannon Entropy}

In the context of signal analysis entropy is a parameter that represents the degree of intrinsic order or degree of organization of the signal: a relatively low entropy value indicates the existence of a structure recognizable in the elementary patterns. Contrarily, a high entropy value indicates the lack of a simple and identifiable structure. In Information Theory the Shannon entropy is defined as in Eq. 3, which we denote as Normalized Shannon Entropy, NSE.

$N S E=-\sum_{j} p_{j} \log \left(p_{j}\right)$; being $\sum_{j} p_{j}=1 ;$ and $p_{j} \log p_{j}=0$ if $p_{j}=0$

where $p_{j}$ is non-negative and has the meaning of a probability. NSE ranges between 0 for information concentrated in a unique point and $\log \mathrm{N}$ for a uniform distribution, where $\mathrm{N}$ is the number of points.

In terms of energy of $\mathrm{AE}$ signals, normalization is required with respect to a total energy. Different normalization strategies were adopted by authors, according to the purpose of research [29, 38-40]. For instance, [40], working with the WT of AE signals in a given frequency band, considered the distribution of WT squared coefficients along the $\mathrm{N}$ points constituting a hit. Then the total energy was considered as the total energy of the frequency band. In [38]

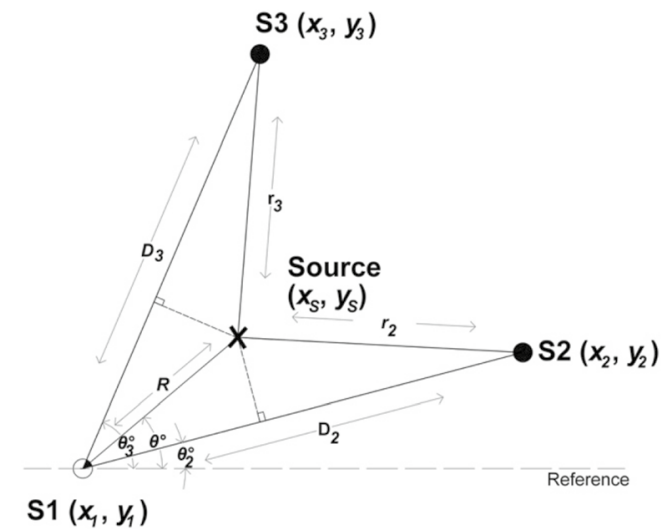

Fig. 4 Three sensors with detection sequence 1, 2 and 3 of source localization technique 
Castro et al. considered the distribution of peak amplitudes in a frequency response signal.

Under some conditions, like described in [41, 42] the non-normalized Shannon Entropy, SE, Eq. 4, is a possible definition.

$S E=-\sum_{j} E_{j} \log \left(E_{j}\right)$

One case is when the total energy of signals is the same. $S E$ is in this case a real number, which takes negative values for energy values greater than 1 , with the convention that " $0 \log (0)$ " $=0$ and where $E_{i}$ is the energy (power) calculated from each raw signal $j$ (measured for example in terms of amplitude voltage, $V$ ) by integrating over the entire record (Eq. 5). The convenience of this definitions lies in the appearance of negative SE values for high energy signals, as a clearer indicator.

$E=\int_{0}^{t} V^{2}(t) d t$

Other case, which is the one considered in this paper, is when the evolution of entropy is considered along each raw signal. Each signal is divided into tranches and the cumulative entropy is calculated along the signal duration. That is to say, the energy distribution in each arriving signal is the required information. The energy distribution of AE transients in the raw signal is more concentrated relatively narrow time intervals. So, cumulative entropy is expected to be lower for authentic AE signals than for noise signals.

\subsection{Application of Cumulative Shannon Entropy to AE Signals}

A modification in the SE analysis over the conventional Eq. 4 is carried out in the present paper. SE in Eq. 4 is a scalar variable obtained from the whole signal. The authors of the present paper propose to study the entire signal in different tranches, identifying each tranche by an index varying from 1 to $n$ the number of analysed samples. In this way, an isolated system is created whenever a new sample arrives (on a cumulative basis) and the entropy is calculated. Taking into account that entropy is directly related to disorder or uncertainty in a system, the main advantage of studying the signal by tranches is that it is possible to know the evolution of entropy as a signal is being generated. The Cumulative Shannon Entropy is defined as Eq. 6:

$\operatorname{CSE}(n)=\sum_{i=1}^{n} S E_{i}$

CSE contains the entropy calculated for the system from the first sample until the n-sample. This is an iterative process whenever a new sample is recorded, being $n$ the number of samples of the signal recorded.

\subsection{Procedure}

\subsubsection{Distinction Between Electrical Noise and Transient AE Signals}

A detailed observation of all the signals and the corresponding CSE curves was performed. Based on this observation, two patterns in CSE curves were found to be qualitatively different: Type (i): a steady growth in value of CSE in which the increase is distributed along the whole signal, and the value is always higher than zero; Type (ii): CSE begins with a value larger than zero but later shows an abrupt change, reaching negative values. These two patterns were assigned to noise and transient AE signals in the present paper according to the physical link to the entropy definition.

\subsubsection{CSE as Indicator of Modes Arrival}

The CSE method is also successful when the time of arrival for different wave modes are studied. From low thickness specimens ( $2 \mathrm{~mm}$ for bar flat and plate), it is deduced that $\mathrm{AE}$ waves propagate as Lamb waves [51]. In a number of preliminary tests, changes in the CSE curve could be observed when different wave modes arrived, that is, the behaviour of cumulative Shannon entropy curve is different for each mode; this means that the modes have different amount of information and can be expressed in different levels of ordering. This fact was used to identify the arrival of different modes, in transient AE signals. For the sake of clarity, the detailed procedure is later explained in detail with some results taken as typical examples.

\subsubsection{Validation of Group Velocity Obtained with CSE}

In the literature, several researchers have proven the effectiveness of analysing very narrow windows at $\mathrm{AE}$ signals to detect the frequency of the Lamb modes [52]. The validation of the detection of S0 and A0 modes can thus be performed by comparing the frequency bands of the AE signals in very narrow windows. Therefore, narrow windows are taken using the arrival time obtained with the CSE.

On the other hand, the calculation of the group velocity for the S0 and A0 modes in the aluminium plate was conducted through the experimental determination of the arrival time as sensors 1 and 2 and the distance between sensors and was compared with the corresponding values obtained through the dispersion curves. A comparison for the effectiveness of CSE and FTC methods for source location for both linear and planar structures was performed. 


\section{Test Model, Experimental Set-Up and Instrumentation}

In order to detect the modes in thin plates and evaluate the time of arrival using the CSE technique, two experimental tests were conducted in two different aluminum specimens using Hsu-Nielsen source. The first test was carried out on a flat bar $2 \mathrm{~mm}$ thick and area $50 \times 1400 \mathrm{~mm}^{2}$. The second test was a plate of square geometry $2 \mathrm{~mm}$ thick and area 300 $\times 300 \mathrm{~mm}^{2}$. In the flat bar, 20 circles of $5 \mathrm{~mm}$ diameter were cut out to simulate discontinuities that affect the propagation path and velocity of the AE waves, a situation that may occur in a real case. Sketches of both specimens can be seen in Fig. 5.

AE signals were acquired with a multichannel system board equipment from Physical Acoustic by using sensors of medium frequency $150 \mathrm{kHz}$ with a $26 \mathrm{~dB}_{\mathrm{AE}}$ gain preamplifier, with sensitivity within the frequency band $100-420 \mathrm{kHz}$, that is to say according to mentioned modes. Sensors were attached on the tested models at the locations shown in Fig. 5. To ensure a good acoustic contact between each of the sensors with the surface of the plate, a silicone grease coupling was used. Subsequently a weight of $0.5 \mathrm{~kg}$ was placed on each of the sensors in order to exert pressure on them. In this way, each of the sensors was perfectly coupled on the surface of the plate. It is a known fact that the effectiveness of a given couplant is dependent on its acoustic impedance, acoustic absorption, application thickness and viscosity. Each of these can have a strong influence on the
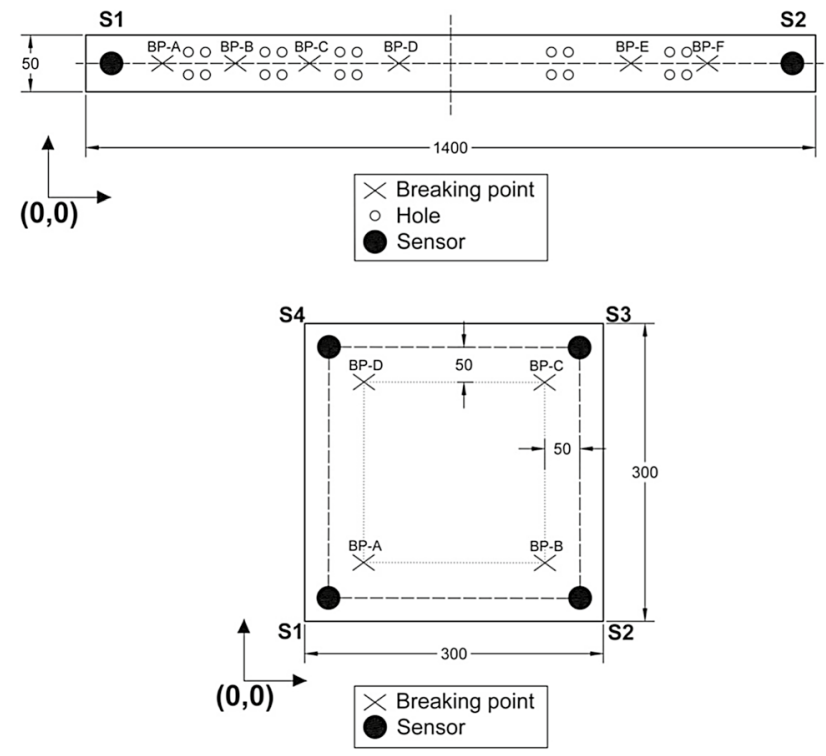

Fig. 5 Schematic of the aluminium test models with AE sensors and breaking points using $\mathrm{H}-\mathrm{N}$. source. Top: flat bar. Distance S1-S2: $1300 \mathrm{~mm}$. Bottom: plate. Distances S1-S2 and S1-S4: $250 \mathrm{~mm}$ sensitivity response of the sensor and can ultimately change the way the sensor responds to different wave modes, but this effect becomes noticeable for frequencies above $400 \mathrm{kHz}$ [53], far beyond the frequency band of the present study. For waveform recording, the sampling frequency was $2.5 \mathrm{MHz}$; the number of samples was 2048 (i.e. one sample per $0.4 \mu$ s) and the pre-trigger was established at 200 samples, after preliminary tests. The threshold was set at $25 \mathrm{~dB}_{\mathrm{AE}}$. Digital filters in the frequency range $100-420 \mathrm{kHz}$ were used during acquisition in order to reject undesirable low and high frequency noises. After mounting the sensors and measuring the background noise in the laboratory, a performance verification test of all the sensors and channels was carried out by using the pencil lead break technique, i.e. the standardized Hsu-Nielsen Source (HSU) [54].

The experimental work involves laboratory experiments with the normalized HSU [55, 56]. Five HSU sources were located at different positions. In the case of the plate, 20 pencil lead breakings were performed in 4 breaking points (BP), from BP-A to BP-D. The locations of breaking points were chosen at $50 \mathrm{~mm}$ inside from where the sensors were located (bottom of the Fig. 5). In the case of the flat bar specimen, arbitrary locations were selected. 30 pencil lead breakings were performed in 6 breaking points, from BP-A to BP-F. The locations can be seen at the top of Fig. 5 .

\section{Results and Discussion}

\subsection{Distinction Between Electrical Noise and Transient AE Signals}

A detailed observation of all the signals and the corresponding CSE curves was performed. Based on this observation, two patterns in CSE curves were found to be qualitatively different: Type (i): a steady growth in value of CSE in which the increase is distributed along the whole signal, and the value is always higher than zero; Type (ii): CSE begins with a value larger than zero but later shows an abrupt change, reaching negative values. Figure 6 shows two examples for Type (i) (Signals 1 and 2) and Type (ii) (Signals 3 and 4) of AE signals recorded in the aluminium plate. These two patterns were assigned to noise and transient AE signals in the present paper according to the physical link to the entropy definition.

\subsection{CSE as Indicator of Modes Arrival}

As it has stated above, CSE was proposed to differentiate between electrical noise and transient signals, both types of signals always appear in any AE test, because for physical reasons noise signals are of higher entropy. The method is also successful when the time of arrival for different 
Fig. 6 Representation of CSE calculated over typical noise and transient AE signals recorded in the aluminium plate. Top: type $i$; bottom: type ii signals. $\mathrm{TH}=25 \mathrm{~dB}_{\mathrm{AE}}$
Signal 1
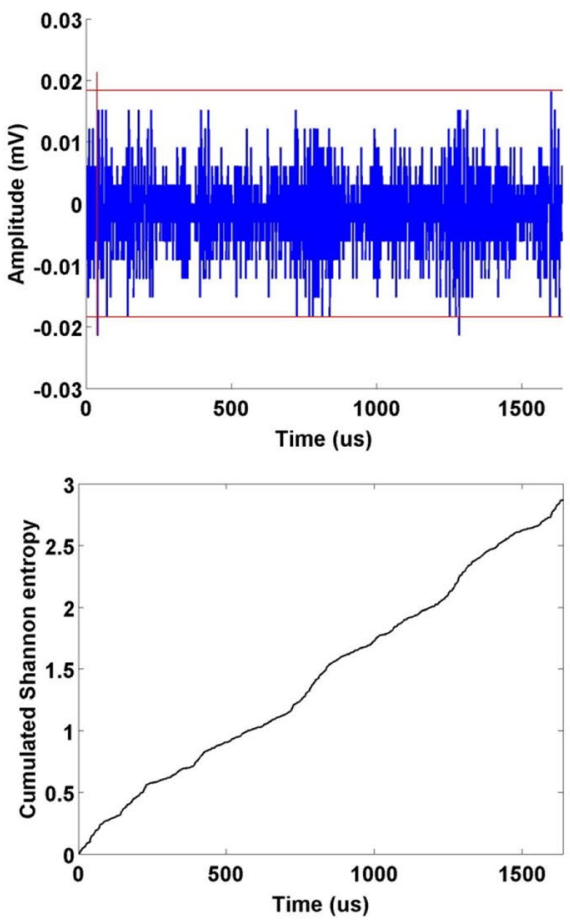

Signal 3
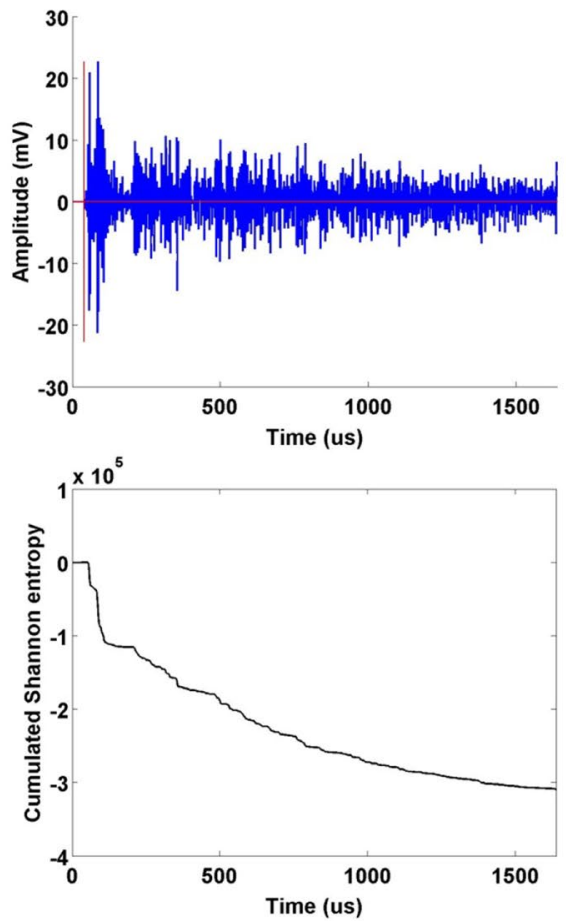

Type (i) signals

Signal 2
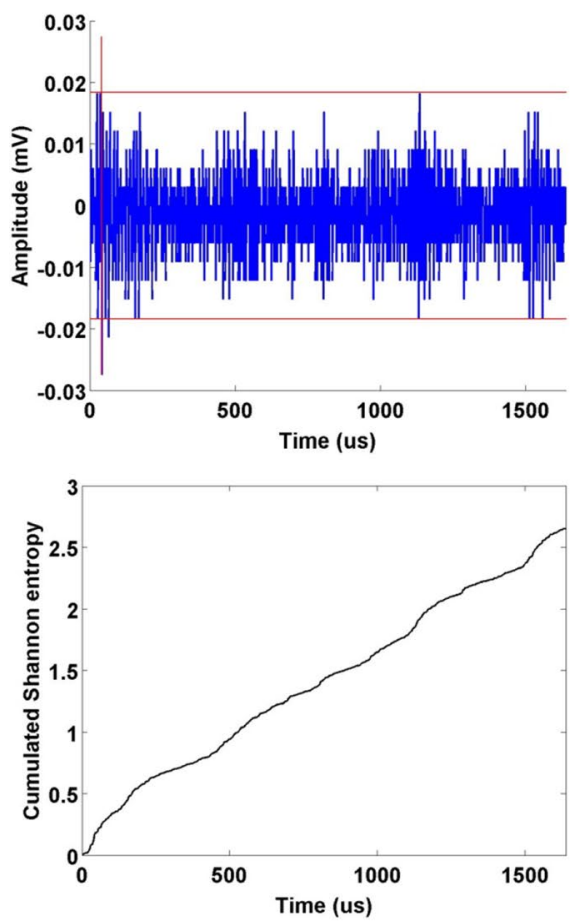

Signal 4
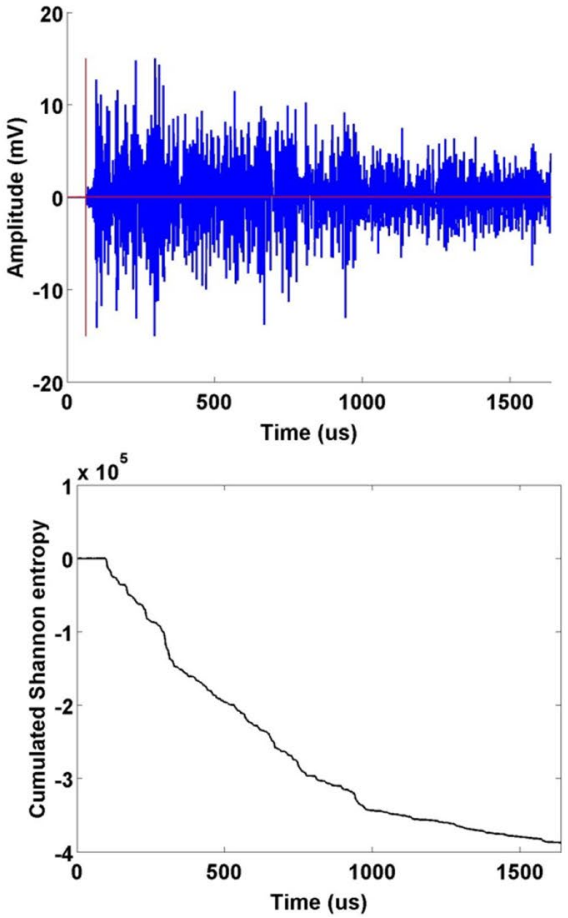

wave modes are studied. From low thickness specimens ( $2 \mathrm{~mm}$ for bar flat and plate), it is deduced that AE waves propagate as Lamb waves [51]. Changes in the CSE curve could be observed when different wave modes arrived, that is, the behaviour of cumulative Shannon entropy curve is different for each mode. This means that the modes have different amount of information and can be expressed in 
different levels of ordering. Figure 7 shows the zoom of two AE signals previously presented in Fig. 6.

As stated in Sect. 4, threshold was set at $25 \mathrm{~dB}_{\mathrm{AE}}$. It was decided by taking into account of the discussion of the Sect. 2.1 in Sect. 2. These signals belong to the same source recorded with sensor 1 (signal 3) and sensor 2 (signal 4) from the Breaking Point A of the aluminium plate. The symmetrical and anti-symmetrical modes can be distinguished in both signals. It is possible to observe that the separation between both modes is higher for signal 4 because sensor 2 is located further from the source (Breaking Point A) compared with the sensor 1 .

A more detailed investigation is carried out in the area of the signal where the S0 and A0 modes are visually identified. For the S0 mode, the study is focused on the beginning of the signal until the instant where the sample of amplitude exceeded the threshold value, and for the A0 mode, the area studied is the part of the signal where the values of amplitude begin to grow faster (arrival of A0 mode). Figures 8 and 9 show a zoom of that part of the signal (blue colour) and the cumulative Shannon entropy curve (black colour) for the S0 and $\mathrm{A} 0$ modes identification, respectively.
It is possible to appreciate a clear growth of the gradient in the CSE curve as the signal ceases to be the random noise of the sensor. The CSE is very sensitive to changes in the samples that are different from zero. This fact is marked with dashed lines and can be seen in Fig. 8. When a sample of the AE signal reaches nonzero value, the CSE curve grows, having its greatest slope just when the S0 mode arrives (39.2 and 64.0 us for signals 3 and 4, respectively). This instant coincides with the true arrival time of the AE signal, being more accurate than the first threshold crossing (40.0 and 64.8 us for signals 3 and 4 , respectively). This growth could express that a higher disorder inside the signal exists when the S0 mode arrives, compared with the previous random electrical noise.

Then, the values of the CSE curve continue to grow until a certain time. If the decline of the CSE curve is observed in the zoom of Fig. 9, it is possible to realize that it coincides with the arrival of the A0 mode for both signals (3 and 4). This occurrence could mean that the system (signal) is being ordered and the process begins when the A0 mode arrives, it can be understood because the predominant mode is more energetic, and the logarithm becomes positive.
Fig. 7 AE signals from the same event recorded at aluminium plate using HSN source. Left: signal 3 (sensor 1); right: signal 4 (sensor 2 ). $\mathrm{TH}=$ $25 \mathrm{~dB}_{\mathrm{AE}}$
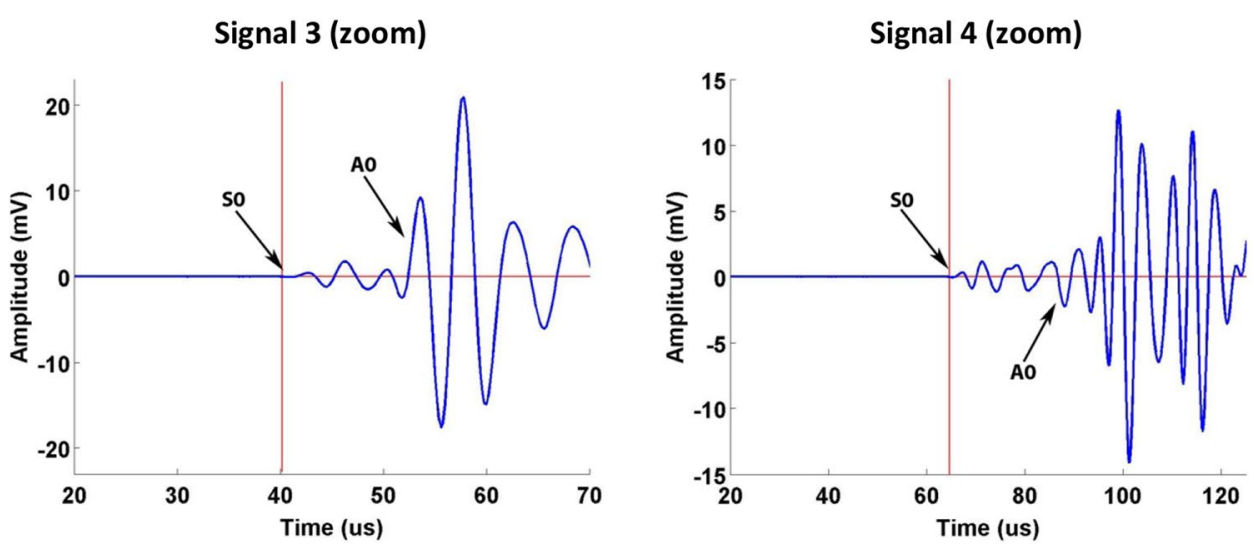

\section{Signal 3}

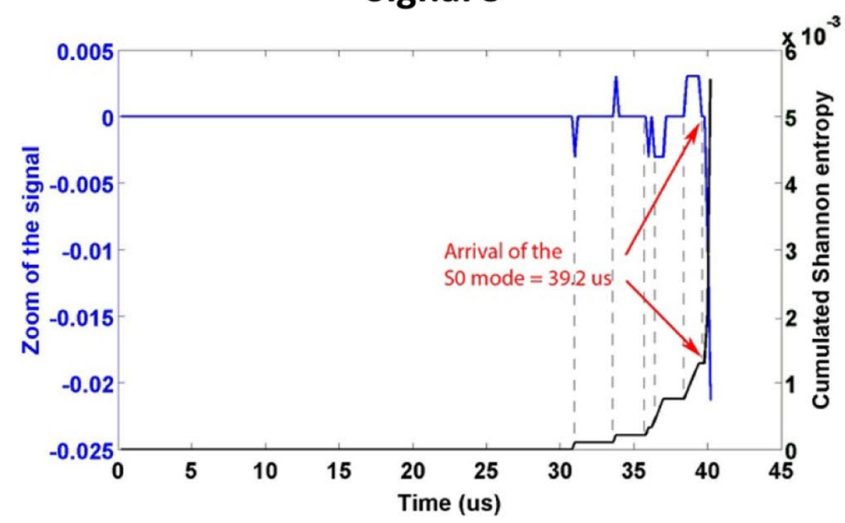

Signal 4

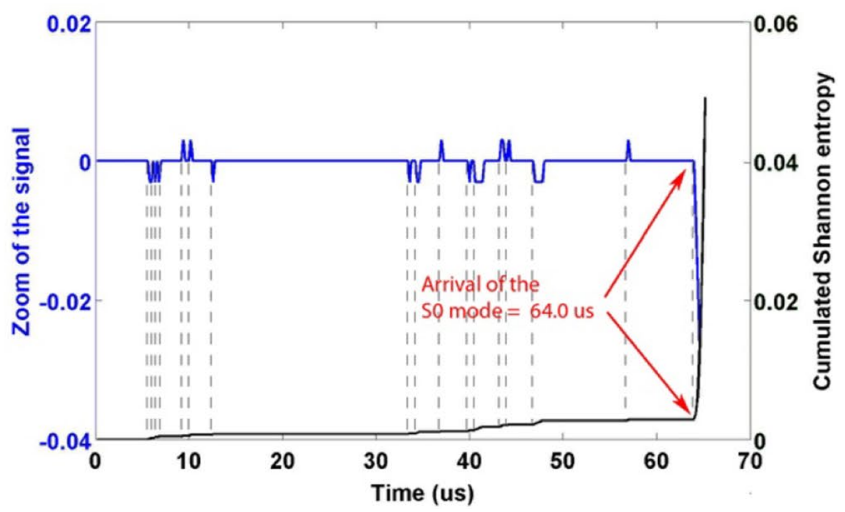

Fig. 8 Part of the signal between the beginning and the first threshold crossing for arrival of the symmetric mode. Left: signal 3 (sensor 1); right: signal 4 (sensor 2). $\mathrm{TH}=25 \mathrm{~dB}_{\mathrm{AE}}$ 


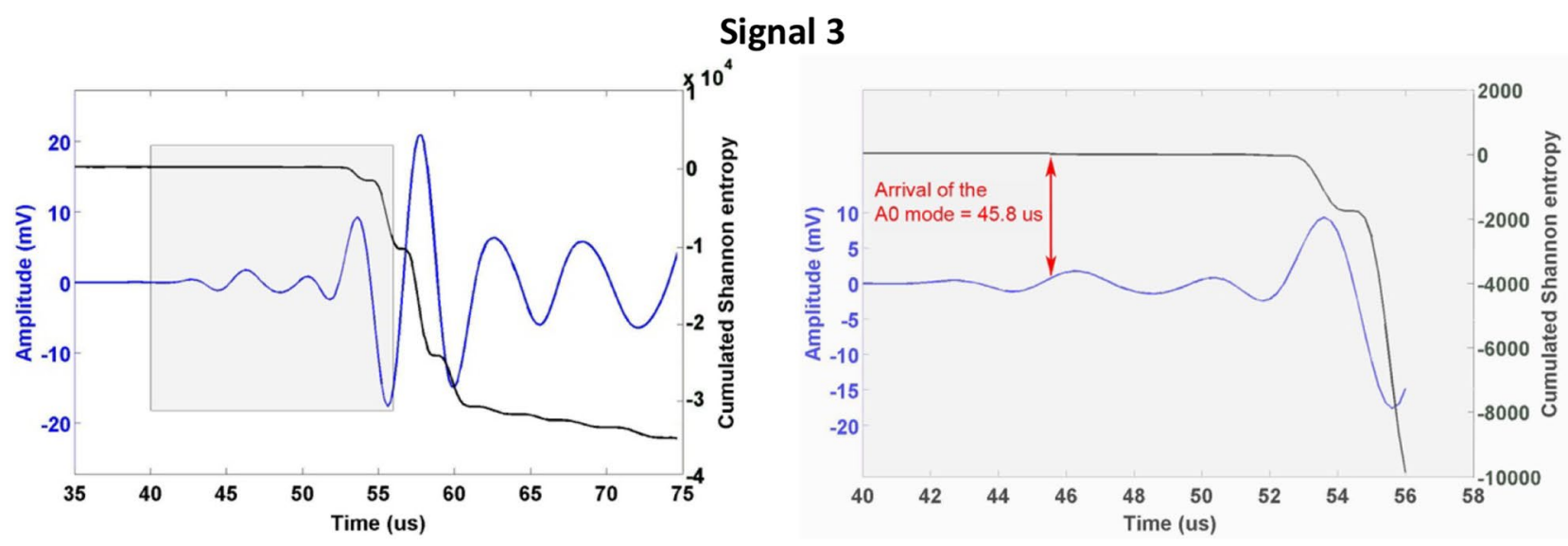

Signal 4
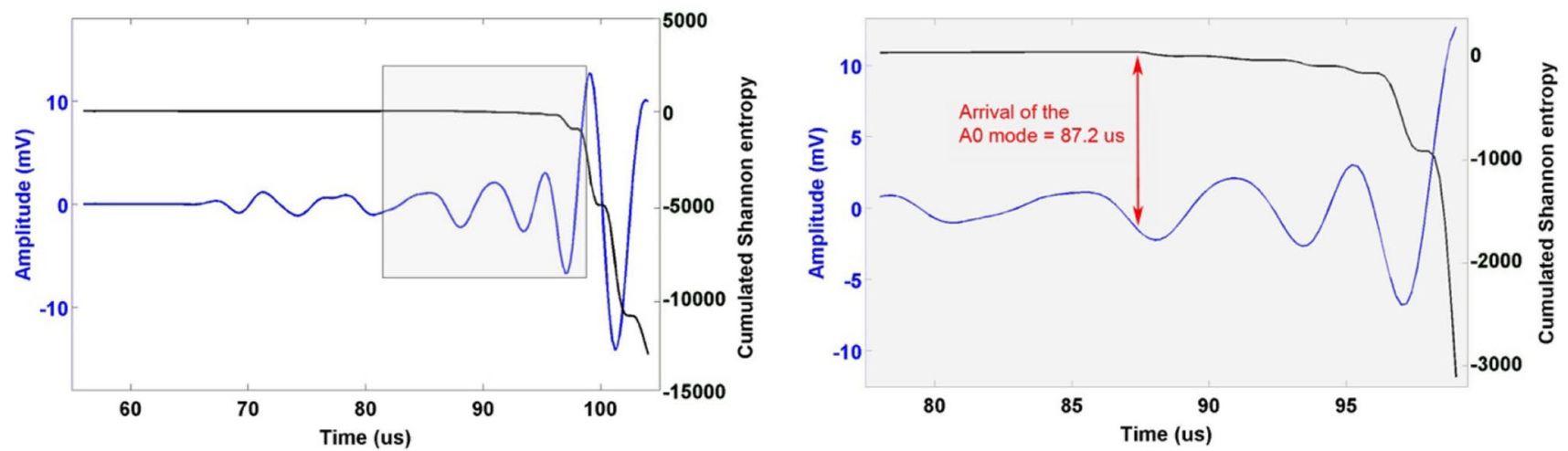

Fig. 9 The arrival of the anti-symmetric mode. Top: signal 3 (sensor 1); bottom: signal 4 (sensor 2). The right figures are zoom of the left ones. $\mathrm{TH}=25 \mathrm{~dB}_{\mathrm{AE}}$

Besides, as it is shown later, the frequency interval range corresponding to A 0 mode is narrower than the one corresponding to S0 mode, which implies less uncertainty. Since the arrival of the A0 mode, the CSE curve does not grow at any ulterior time. This observation means the A0 mode has less information (less entropy) because fewer parameters are needed to parameterize this mode. It is noted that when the curve descends, CSE takes greater absolute values than those present in the ascending values of the first mode S0, so it is possible to realise that it is the predominant mode of the signal, the most energetic and with higher amplitude. These observations occur in all registered signals in both aluminium specimens.

\subsection{Experimental Group Velocity Group Using Normalized HSU Source}

In the literature, several researchers have proven the effectiveness of analysing very narrow windows at $\mathrm{AE}$ signals to detect the frequency of the Lamb modes [52]. The validation of the detection of $\mathrm{S} 0$ and $\mathrm{A} 0$ modes can thus be performed by comparing the frequency bands of the AE signals in very narrow windows. Therefore, narrow windows are taken using the arrival time obtained with the CSE. Table 1 shows the time of arrival (TOA) of the AE signal obtained with CSE for both modes in the aluminium plate as shown in Figs. 8 and 9.

The calculation of the group velocity for the S0 and A0 modes in the aluminium plate is conducted through the data obtained in Table 1. Based on the relation of $\Delta S$ and $\Delta T$ between sensors 1 and 2, the velocity can be obtained for each mode as follows:

Table 1 TOA obtained with CSE for both modes in the aluminium plate and distances of the sensors to the breaking point A

\begin{tabular}{llll}
\hline & $\mathrm{S} 0(\mu \mathrm{s})$ & $\mathrm{A} 0(\mu \mathrm{s})$ & $\begin{array}{l}\text { Distance } \\
\text { from BP1 } \\
(\mathrm{cm})\end{array}$ \\
\hline $\begin{array}{l}\text { 1st signal of the event } \\
(\text { sensor 1-signal 3) }\end{array}$ & 39.8 & 45.8 & 5.292 \\
$\begin{array}{l}\text { 2nd signal of the } \\
\text { event (sensor 2- } \\
\text { signal 4) }\end{array}$ & 64.0 & 87.2 & 19.708 \\
\hline
\end{tabular}


$V_{S 0}=\frac{14.416 \mathrm{~cm}}{(64-39.8) \mu s}=0.59 \mathrm{~cm} / \mu s$

$V_{A 0}=\frac{14.416 \mathrm{~cm}}{(87.2-45.8) \mu s}=0.34 \mathrm{~cm} / \mu s$

Figure 10 shows the frequency spectrum for different windows in the first and second signals of the event (signals 3 and 4 previously showed). For the signal 3 the windows are (37-43) and (43-49) $\mu$ s for S0 and A0 modes, respectively. On the other hand, for signal 4 , the windows are (61-67) $\mu$ s for $S 0$ and (84-90) $\mu$ s for A0 modes. Note that the arrival time of each mode (detected with the novel CSE) coincides with the average width of the window where this is calculated.

If a Lamb wave is analysed at a certain distance after propagation, it is possible to see how the peaks of frequency for $\mathrm{S} 0$ and $\mathrm{A} 0$ modes are different from each other. It can be seen from Fig. 10 that the peaks of frequency for S0 and A0 modes could be detected at the time of arrival with CSE. For signal 3, located closer to the source, the peaks of frequency are 300 and $250 \mathrm{kHz}$ for modes S0 and A0, respectively. In the case of signal 4, peaks of 280 and $250 \mathrm{kHz}$ are observed for $\mathrm{S} 0$ and $\mathrm{A} 0$ modes, respectively. It is found that the dispersion is higher for $\mathrm{S} 0$ than that for $\mathrm{A} 0$ mode in the aluminium plate. This fact has been confirmed by other researchers [29], and it can be seen that the peak of frequency for $\mathrm{S} 0$ mode is displaced in signal 4 with respect to signal 3 (it is recorded at greater distance). This frequency deviation indicates the existence of dispersion.

The theoretical calculation of the group velocity in the aluminium plate is performed using the following data (Fig. 11): longitudinal velocity: $C_{L}=6.320 \mathrm{~mm} / \mu \mathrm{s}$, shear velocity: $C_{S}=3.260 \mathrm{~mm} / \mu$ s and thickness $=2 \mathrm{~mm}$ [57]. If the velocity of each mode is observed at the dispersion curves in Fig. 11, it is realised that: For S0 mode at $300 \mathrm{kHz}$, the velocity obtained at windows of the signal 3 (37-43) $\mu$ s and signal 4 (61-67) $\mu$ s is $5.6 \mathrm{~m} / \mathrm{ms}$; for A0 mode at $250 \mathrm{kHz}$, the velocity obtained at windows of the signal 3 (43-49) $\mu$ s and $4(84-90) \mu s$ is $3.2 \mathrm{~m} / \mathrm{ms}$. Table 2 shows the comparison between the experimental values obtained from

\section{Signal 3}
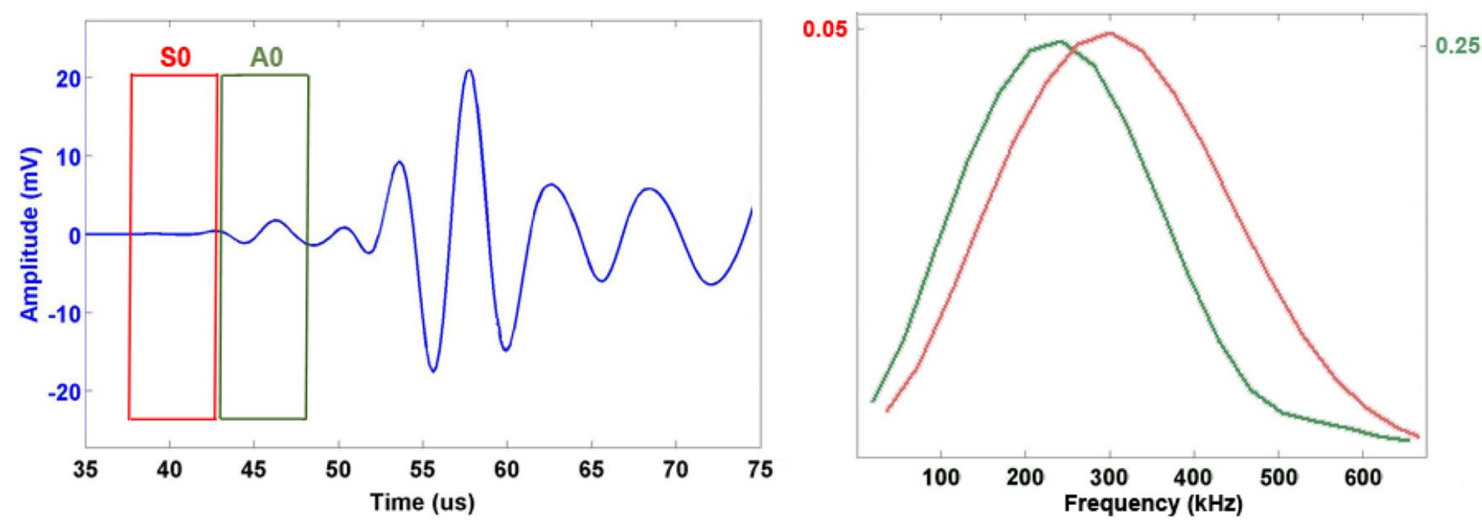

Signal 4
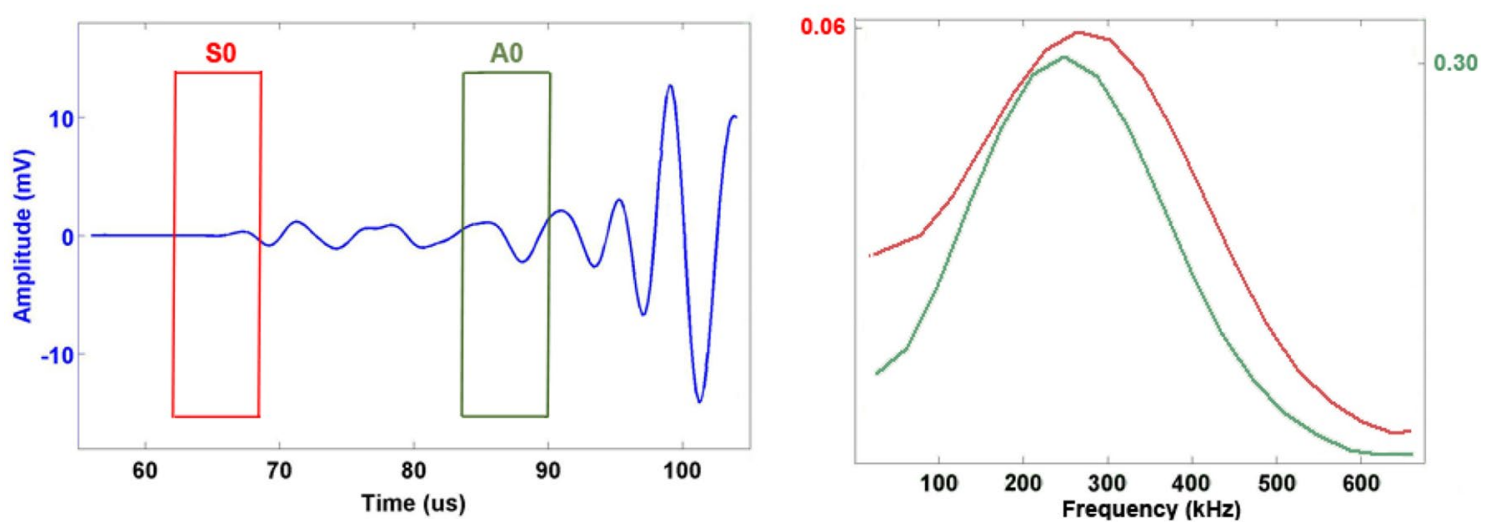

Fig. 10 Left: Narrow windows marked over AE signals 3. Right: Spectral response obtained from different time windows for S0 and A0 modes of signal 3 and signal 4) 
Fig. 11 Theoretical group velocity in an aluminium plate calculated for $2 \mathrm{~mm}$ of thickness

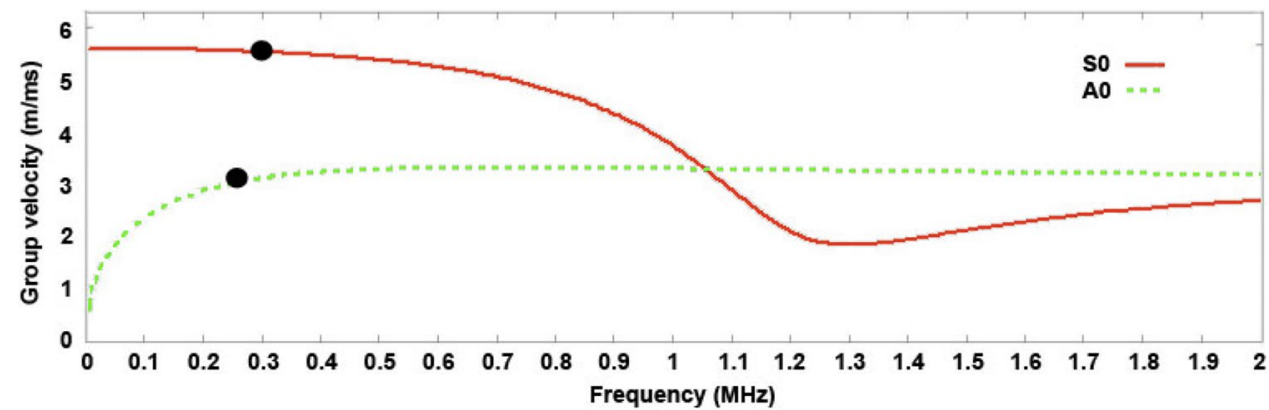

Table 2 Theoretical and experimental group velocity measured in an aluminium plate

\begin{tabular}{lll}
\hline Method & S0 mode $(\mathrm{m} / \mathrm{ms})$ & $\begin{array}{l}\text { A0 } \\
\text { mode } \\
(\mathrm{m} / \mathrm{ms})\end{array}$ \\
\hline Theoretical & 5.6 & 3.2 \\
Experimental & 5.9 & 3.4 \\
\hline
\end{tabular}

Eqs. 7 and 8, and the theoretical values shown in Fig. 10. It is noticed that these values are very similar to each other. This observation confirms that the arrival of S0 and A0 modes could be detected with the CSE.

\subsection{CSE Applied to Detection of TOA in Order to Improve the Localization of AE Sources}

The localization of AE sources in this work is performed using the minimum number of sensors and it is not the purpose of this work to use an over-determined system. It has been shown that the threshold-crossing technique produces errors to locate AE sources (Figs. 2 and 3) when the first threshold crossing is used as time of arrival (TOA $\left.{ }_{\mathrm{FTC}}\right)$. In this section, the CSE has been developed to detect the first mode of arrival (S0) for AE signals recorded in a flat bar and plate, that is, $\mathrm{TOA}_{\mathrm{CSE}}$. To verify the improvement that this new method offers in the localization of $\mathrm{AE}$ sources, the comparison between $\mathrm{TOA}_{\mathrm{FTC}}$ and $\mathrm{TOA}_{\mathrm{CSE}}$ is performed using linear and planar structures.

\subsubsection{Linear Structure}

A Matlab code for a linear structure is developed to determine the location of the breaking points in a flat bar. The $\mathrm{AE}$ signals are set in events to determine the difference of time between the signals that arrive to $\mathrm{S} 1$ and $\mathrm{S} 2$. Equation 1 is applied using the difference of time obtained for FTC $\left(\mathrm{TOA}_{\mathrm{FTC}}\right)$ and CSE $\left(\mathrm{TOA}_{\mathrm{CSE}}\right)$. Relative errors for both methods are calculated and results are shown in Table 3. The results displayed in Table 3 reveal that the errors are in general lower for CSE method $\left(\mathrm{TOA}_{\mathrm{CSE}}\right)$ than those for FTC $\left(\mathrm{TOA}_{\mathrm{FTC}}\right)$ with 27 out of the 30 times that the Hsu-Nielsen source tests are carried out, CSE outperforms the FTC. Therefore, the novel CSE is an improved method that can be used for detecting the time of arrival of the AE signals and thus, to improve the localization of AE sources. Figure 12 shows the average relative error calculated based on 5 lead breaks in each breaking point. It has been shown that CSE method produces lower errors in all cases.

\subsubsection{Planar Structure}

A Matlab code for a planar structure is developed to determine the location of the breaking points in a plate. The AE signals are set in events to determine the difference of time among the 3 sensors. Thereby, two parabolas could be obtained and the intersection of them is identified to locate the HSU sources. Equation 2 is applied using the difference of time obtained for FTC $\left(\mathrm{TOA}_{\mathrm{FTC}}\right)$ and CSE $\left(\mathrm{TOA}_{\mathrm{CSE}}\right)$ methods. The relative errors for both methods are calculated and results are shown in Table 4. Similar to the results obtained from flat bar, the results for planar structure obtained from the plate and shown in Table 4 reveal that the errors are much lower for CSE method than those for FTC in most cases where the Hsu-Nielsen source is used.

There are small errors occurring in the experiments with flat bar and plate. This could be originated by many factors, mainly due to the deterioration of the waves in their way from the source to the sensor, the use of a unique propagation velocity in all directions or the dispersion of propagation modes which depends on the frequency (as shown in Fig. 10). All these factors cause further difficulties in determining the time of arrival $[6,9,44]$.

Figure 13 shows the average relative error calculated based on 5 lead breaks in each breaking point. The same results are observed where the errors produced by CSE are lower than those from the FTC in all cases. Therefore, the novel CSE method is an improved method that can be used for estimating the time of arrival of the AE signals and thus, it provides better results for the localization of AE sources. 
Table 3 Comparison of time of arrival between FTC and CSE methods for the transient $\mathrm{AE}$ signals recorded in a flat bar

\begin{tabular}{|c|c|c|c|c|c|c|}
\hline $\begin{array}{l}\text { Breaking } \\
\text { point }\end{array}$ & $\begin{array}{l}\text { Number of } \\
\text { lead }\end{array}$ & $\begin{array}{l}\text { Real coordi- } \\
\text { nate }(\mathrm{cm})\end{array}$ & $\begin{array}{l}\text { TOA }_{\text {FTC }} \text { coordi- } \\
\text { nate }(\mathrm{cm})\end{array}$ & $\begin{array}{l}\text { Relative } \\
\text { error }(\%)\end{array}$ & $\begin{array}{l}\text { TOA }_{\mathrm{CSE}} \text { coordi- } \\
\text { nate }(\mathrm{cm})\end{array}$ & $\begin{array}{l}\text { Relative } \\
\text { error } \\
(\%)\end{array}$ \\
\hline \multirow[t]{5}{*}{ A } & 1 & 3.0 & 2.17 & 1.63 & 2.90 & 0.32 \\
\hline & 2 & 3.0 & 2.14 & 1.69 & 2.70 & 0.67 \\
\hline & 3 & 3.0 & 1.42 & 2.98 & 2.16 & 1.65 \\
\hline & 4 & 3.0 & 2.13 & 1.69 & 2.85 & 0.40 \\
\hline & 5 & 3.0 & 2.12 & 1.72 & 2.85 & 0.40 \\
\hline \multirow[t]{5}{*}{ B } & 1 & 21.2 & 20.91 & 0.89 & 21.43 & 0.52 \\
\hline & 2 & 21.2 & 20.99 & 0.67 & 21.73 & 1.31 \\
\hline & 3 & 21.2 & 20.84 & 1.07 & 21.78 & 1.44 \\
\hline & 4 & 21.2 & 20.51 & 1.95 & 21.04 & 0.54 \\
\hline & 5 & 21.2 & 20.48 & 2.02 & 21.24 & 0.01 \\
\hline \multirow[t]{5}{*}{$\mathrm{C}$} & 1 & 35.3 & 34.35 & 4.42 & 35.34 & 0.10 \\
\hline & 2 & 35.3 & 34.37 & 4.30 & 35.39 & 0.11 \\
\hline & 3 & 35.3 & 34.35 & 4.42 & 35.34 & 0.10 \\
\hline & 4 & 35.3 & 34.34 & 4.46 & 35.34 & 0.10 \\
\hline & 5 & 35.3 & 34.33 & 4.50 & 35.34 & 0.10 \\
\hline \multirow[t]{5}{*}{ D } & 1 & 43.4 & 43.00 & 2.95 & 43.51 & 0.42 \\
\hline & 2 & 43.4 & 42.57 & 5.77 & 43.31 & 0.88 \\
\hline & 3 & 43.4 & 43.00 & 2.95 & 43.46 & 0.10 \\
\hline & 4 & 43.4 & 43.03 & 2.76 & 43.51 & 0.42 \\
\hline & 5 & 43.4 & 42.90 & 3.59 & 43.46 & 0.10 \\
\hline \multirow[t]{5}{*}{$\mathrm{E}$} & 1 & 91.9 & 92.36 & 1.43 & 91.59 & 0.89 \\
\hline & 2 & 91.9 & 92.82 & 2.79 & 91.98 & 0.28 \\
\hline & 3 & 91.9 & 92.24 & 1.05 & 91.44 & 1.33 \\
\hline & 4 & 91.9 & 92.40 & 1.54 & 91.49 & 1.19 \\
\hline & 5 & 91.9 & 92.35 & 1.40 & 91.59 & 0.89 \\
\hline \multirow[t]{5}{*}{$\mathrm{F}$} & 1 & 99.9 & 100.93 & 2.34 & 100.19 & 0.56 \\
\hline & 2 & 99.9 & 101.00 & 2.51 & 100.24 & 0.68 \\
\hline & 3 & 99.9 & 100.88 & 2.22 & 100.14 & 0.44 \\
\hline & 4 & 99.9 & 100.93 & 2.34 & 100.19 & 0.56 \\
\hline & 5 & 99.9 & 101.05 & 2.63 & 100.29 & 0.80 \\
\hline
\end{tabular}

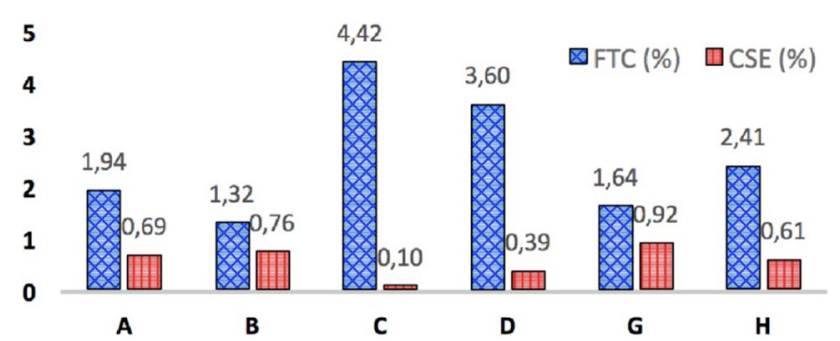

Fig. 12 Comparison of relative error between FTC and CSE methods for the breaking points A-H using Hsu-Nielsen source performed in an aluminium flat bar

\section{Conclusions}

A new procedure to identify and detect Lamb waves using the AE technique has been presented for thin aluminium specimens. It involves an algorithm based on cumulative Shannon entropy which is able to distinguish between electrical noise and transient AE signals and make a suitable separation between the $\mathrm{S} 0$ and $\mathrm{A} 0$ Lamb modes in the AE signals. The method has been validated using the normalized Hsu Nielsen source in an aluminium flat bar and plate. Cumulative Shannon entropy curve shows a different trend between recorded AE information. For transient signals, the CSE curve shows a significant decrease. However, for the electrical noise, the CSE curve is always increasing. Moreover, cumulative Shannon entropy displays very different behaviour for each of the A0 and S0 modes of AE transient signals, thus allowing the identification of these modes. Firstly, an increase of cumulative Shannon entropy curve at the arrival of S0 mode indicates that there is higher uncertainty in information, which means, higher entropy (higher disorder). However, the arrival of A0 mode shows a steady decrease in cumulative entropy 
Table 4 Comparison of time of arrival between FTC and CSE methods for the transient $\mathrm{AE}$ signals recorded in a plate

\begin{tabular}{|c|c|c|c|c|c|c|c|c|c|c|c|}
\hline \multirow[t]{2}{*}{$\begin{array}{l}\text { Break- } \\
\text { ing point }\end{array}$} & \multirow[t]{2}{*}{$\begin{array}{l}\text { Number } \\
\text { of lead }\end{array}$} & \multicolumn{2}{|c|}{$\begin{array}{l}\text { Real coordi- } \\
\text { nate }(\mathrm{cm})\end{array}$} & \multicolumn{2}{|c|}{$\begin{array}{l}\text { TOA }_{\text {FTC }} \text { coordi- } \\
\text { nate }(\mathrm{cm})\end{array}$} & \multicolumn{2}{|c|}{$\begin{array}{l}\text { Relative error } \\
(\%)\end{array}$} & \multicolumn{2}{|c|}{$\begin{array}{l}\text { TOA }_{\mathrm{CSE}} \text { coordi- } \\
\text { nate }(\mathrm{cm})\end{array}$} & \multicolumn{2}{|c|}{$\begin{array}{l}\text { Relative error } \\
(\%)\end{array}$} \\
\hline & & X & $\mathrm{Y}$ & $X$ & $\mathrm{Y}$ & $X$ & Y & $X$ & $\mathrm{Y}$ & $X$ & $\mathrm{Y}$ \\
\hline \multirow[t]{5}{*}{ A } & 1 & 5.1 & 5.1 & 5.26 & 4.85 & 3.11 & 4.97 & 5.15 & 5.27 & 0.92 & 3.33 \\
\hline & 2 & 5.1 & 5.1 & 5.34 & 4.76 & 4.79 & 6.71 & 5.21 & 5.16 & 2.10 & 1.18 \\
\hline & 3 & 5.1 & 5.1 & 4.18 & 5.58 & 18.04 & 9.50 & 4.28 & 5.15 & 16.09 & 0.98 \\
\hline & 4 & 5.1 & 5.1 & 5.73 & 4.98 & 12.35 & 2.35 & 5.63 & 5.20 & 10.36 & 1.96 \\
\hline & 5 & 5.1 & 5.1 & 5.58 & 4.83 & 9.38 & 5.39 & 5.58 & 5.15 & 9.38 & 0.98 \\
\hline \multirow[t]{5}{*}{ B } & 1 & 19.05 & 5.05 & 19.99 & 4.83 & 4.94 & 4.35 & 19.20 & 5.02 & 0.79 & 0.58 \\
\hline & 2 & 19.05 & 5.05 & 20.06 & 4.85 & 5.30 & 3.96 & 19.56 & 5.06 & 2.68 & 0.18 \\
\hline & 3 & 19.05 & 5.05 & 20.16 & 4.75 & 5.83 & 5.95 & 19.64 & 4.44 & 3.10 & 11.98 \\
\hline & 4 & 19.05 & 5.05 & 20.05 & 5.04 & 5.24 & 0.20 & 19.21 & 5.23 & 0.84 & 3.54 \\
\hline & 5 & 19.05 & 5.05 & 20.03 & 4.97 & 5.14 & 1.58 & 19.33 & 5.17 & 1.47 & 2.28 \\
\hline \multirow[t]{5}{*}{$\mathrm{C}$} & 1 & 20 & 19.9 & 20.38 & 20.13 & 1.89 & 1.13 & 20.38 & 20.13 & 1.89 & 1.13 \\
\hline & 2 & 20 & 19.9 & 20.90 & 19.93 & 4.50 & 0.14 & 20.90 & 19.93 & 4.50 & 0.14 \\
\hline & 3 & 20 & 19.9 & 20.90 & 19.93 & 4.50 & 0.14 & 20.47 & 20.04 & 2.34 & 0.69 \\
\hline & 4 & 20 & 19.9 & 20.18 & 20.64 & 0.88 & 3.70 & 19.91 & 20.52 & 0.44 & 3.12 \\
\hline & 5 & 20 & 19.9 & 20.16 & 20.58 & 0.80 & 3.40 & 20.34 & 20.39 & 1.70 & 2.47 \\
\hline \multirow[t]{5}{*}{$\mathrm{D}$} & 1 & 5.1 & 19.9 & 5.30 & 20.59 & 3.91 & 3.46 & 5.30 & 20.59 & 3.91 & 3.46 \\
\hline & 2 & 5.1 & 19.9 & 5.83 & 20.77 & 14.25 & 4.35 & 5.60 & 20.76 & 9.81 & 4.30 \\
\hline & 3 & 5.1 & 19.9 & 5.27 & 20.71 & 3.27 & 4.08 & 4.93 & 20.67 & 3.32 & 3.87 \\
\hline & 4 & 5.1 & 19.9 & 5.32 & 20.53 & 4.23 & 3.15 & 4.91 & 20.34 & 3.77 & 2.19 \\
\hline & 5 & 5.1 & 19.9 & 5.20 & 20.96 & 1.99 & 5.33 & 5.36 & 20.81 & 5.04 & 4.57 \\
\hline
\end{tabular}

10

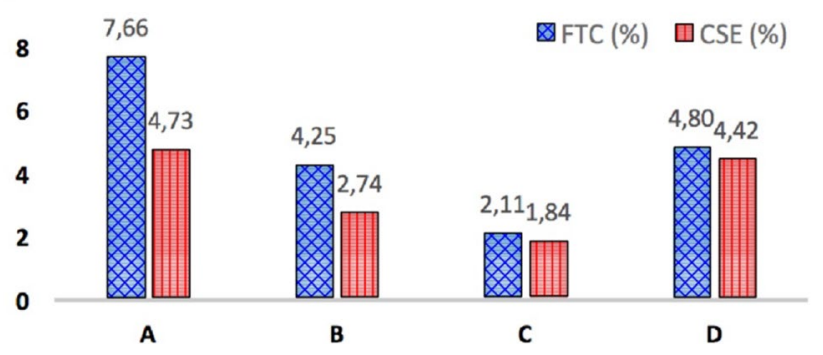

Fig. 13 Comparison of relative error between FTC and CSE methods for the breaking points A-D using Hsu-Nielsen source performed in an aluminium plate

expressing that the signals began to order because A0 mode is the predominant, the most energetic, with higher amplitude and with narrower involved frequency interval. Secondly, the use of very narrow windows allows one to know the frequency range, velocity and dispersion curves, and thereby, validate experimentally the detection of Lamb modes performed with cumulative Shannon entropy.

The use of Shannon entropy on a cumulative basis is an original contribution of this paper and has improved the detection of the arrival of S0 and A0 Lamb modes. Moreover, in order to verify the method's sensitivity in the detection of the first mode of arrival, a localization of $\mathrm{AE}$ signals is performed in both specimens. The comparison of the estimated time of arrival based on first threshold crossing and cumulative Shannon entropy is performed. The studies in both specimens presented here confirm the accuracy and feasibility of the cumulative Shannon entropy-based localization. The results are significantly improved when the onset of signal is taken with the cumulative Shannon entropy, and can be effectively used to find the source location. The errors could be mainly attributed to dispersion of modes in Lamb waves and the deterioration of the signals. The proposed method for AE source localization provides a new alternative in the identification process of wave modes and allows more accurate damage localization.

Acknowledgements R.P acknowledges the University of Buenos Aires for the Project 20020160100038BA.

Open Access This article is distributed under the terms of the Creative Commons Attribution 4.0 International License (http://creativeco mmons.org/licenses/by/4.0/), which permits unrestricted use, distribution, and reproduction in any medium, provided you give appropriate credit to the original author(s) and the source, provide a link to the Creative Commons license, and indicate if changes were made. 


\section{References}

1. Gopalakrishnan, S., Ruzzene, M., Hanagud, S.: Computational techniques for structural health monitoring. Springer, London (2013)

2. Laodeno, R.N., Yoshida, K.: Non-destructive testing technique. LAP Lambert Academic Publishing, Saarbrücken (2013)

3. Fang, Y., Wen, L., Tee, K.F.: Reliability analysis of repairable k-out-of-n system from time response under several times stochastic shocks. Smart Struct. Syst. 14(4), 559-567 (2014)

4. Tee, K.F., Cai, Y., Chen, H.P.: Structural damage detection using quantile regression. J. Civil Struct. Health Monit. 3(1), 19-31 (2013)

5. Klepka, A., Staszewski, W.J., DiMaio, D., Scarpa, F., Tee, K.F., Uhl, T.: Sensor location analysis in nonlinear acoustics used for damage detection in composite chiral sandwich panels. Adv. Sci. Technol. 83, 223-231 (2013)

6. Hardy, JR.: Acoustic emission, microseismic activity. Principles, techniques, and geotechnical application, 1, 292. A.A. Balkena Publ. USA (2003)

7. Jenal, R.B., Staszewski, W.J.; Scarpa, F.; Tee, K.F. Damage detection in smart chiral sandwich structures using nonlinear acoustics. In: Proceedings of the 20th International Conference on Adaptive Structures and Technologies, Hong Kong, China, 20-22 October, 2009.

8. Klepka, A., Staszewski, W.J., Uhl, T., DiMaio, D., Scarpa, F., Tee, K.F.: Impact damage detection in composite chiral sandwich panels. Key Eng. Mater. 518, 160-167 (2012)

9. Miller, K.R., Hill, E.K.: Non-Destructive Testing Handbook, Acoustic Emission Testing. American Society for Non-Destructive Testing, Columbus (2005)

10. Sagasta, F., Benavent-Climent, A., Fernández-Quirante, T., Gallego, A.: Modified Gutenberg-Richter coefficient for damage evaluation in reinforced concrete structures subjected to seismic simulations on a shaking table. J. Nondestr. Eval. 33(4), 616-631 (2014)

11. Wevers, M., Lambrighs, K.: Applications of acoustic emission for SHM: a review. Encyclopedia of structural health monitoring. Wiley, New York (2009)

12. Wevers, M.: Listening to the sound of materials: acoustic emission for the analysis of material behavior. NDT\&E Int. 30, 99-106 (1997)

13. Flynn, E.B., Todd, M.D., Wilcox, P.D., Drinkwater, B.W., Croxford, A.J.: Maximum-likelihood estimation of damage location in guided-wave structural health monitoring. Proc. R. Soc. A 467, 2575-2596 (2011)

14. Courtier, M.R., Croxford, A.J., Atherton, K.: Guided wave propagation modelling to aid understanding of acoustic emission system performance on complex aerospace structures, 8th European workshop on structural health monitoring (EWSHM 2016), 5-8 July 2016, Spain, Bilbao

15. Al-Jumaili, S.K.H., Pearson, M.R., Holford, K.M., Eaton, M.J., Pullin, R.: Acoustic emission source location in complex structures using full automatic delta T mapping technique. Mech. Syst. Signal Process. 72-73, 513-524 (2016)

16. Sedlak, P., Hirose, Y., Enoki, M.: Acoustic emission localization in thin multi-layer plates using first-arrival determination. Mech. Syst. Signal Process. 36, 636-649 (2013)

17. Allen, R.: Automatic phase pickers: their present use and future prospects. Bull. Seismol. Soc. Am. 72, 225-242 (1982)

18. Hensman, J., Pullin, R., Eaton, M., Worden, K., Holford, K.M., Evans, S.L.: Detecting and identifying artificial acoustic emission signals in an industrial fatigue environment. Meas. Sci. Technol. 20(4), 045101 (2009)
19. Eaton, M.J., Pullin, R., Holford, K.M.: (1) Towards improved damage location using acoustic emission. Proc. IMechE Part C: J. Mech. Eng. Sci. 226(9), 2141-2153 (2012)

20. Eaton, M.J., Pullin, R., Holford, K.M.: (2) Acoustic emission source location in composite materials using Delta T Mapping. Compos. Part A: Appl. Sci. Manuf. 43, 856-863 (2012)

21. Dehghan, N.E., Farhidzadeh, A., Salamone, S.: Determination of the probability zone for acoustic emission source location in cylindrical shell structures. Mech. Syst. Signal Process. 60-61, 971-985 (2015)

22. Dai, H., MacBeth, C.: Automatic picking of seismic arrivals in local earthquake data using an artificial neural network. Geophys. J. Int. 120, 758-774 (1995)

23. Maeda, N.: A method for reading and checking phase times in auto-processing system of seismic wave data. Zisin1/4Jishin 38 365-379 (1985)

24. Sedlak, P., Hirose, Y., Khan, S.A., Enoki, M., Sikula, J.: New automatic localization technique of acoustic emission signals in thin metal plates. Ultrasonics 49, 254-262 (2009)

25. Ziola, S.M., Gorman, M.R.: Source location in thin plates using cross-correlation. J. Acoust. Soc. Am. 90, 2551-2556 (1991)

26. Jiao, J., He, C., Wu, B., Fei, R., Wang, X.: Application of wavelet transform on modal acoustic emission source location in thin plates with one sensor. Int. J. Press. Vessel. Pip. 81, 427-431 (2004)

27. Kundu, T., Yang, X., Nakatani, H., Takeda, N.: A two-step hybrid technique for accurately localizing acoustic source in anisotropic structures without knowing their material properties. Ultrasonics 56, 271-278 (2015)

28. Tsaugouri, E., Aggelis, D.G.: The influence of sensor size on acoustic emission waveforms-a numerical study. Appl. Sci. 8, 168 (2018). https://doi.org/10.3390/app8020168

29. Suarez, E., Roldán, A., Gallego, A., Benavent-Climent, A.: Entropy analysis for damage quantification of hysteretic dampers used as seismic protection of buildings. Appl. Sci. 7, 628 (2017)

30. Gary, J., Hamstad, M.A.: On the far-field structure of waves generated by a pencil lead break on a thin plate. J. Acoust. Emiss. 12, 157-170 (1994)

31. Prosser, W.H., Hamstad, M.A., Gary, J., Gallagher, A.O.: Finite element and plate theory modeling of acoustic emission waveforms. J. Nondestr. Eval. 18, 83-90 (1999)

32. Chen, J., Li, G.: Wavelet entropy and its application in power signal analysis. Entropy 16, 3009-3025 (2014). https://doi. org/10.3390/e16063009

33. Martin, N.F.G., England, J.W.: Mathematical Theory of Entropy. Cambridge University Press, Cambridge (2011)

34. Baez, J.C., Fritz, T., Leinster, T.: A characterization of entropy in terms of information loss. Entropy 13, 1945-1957 (2011)

35. Hnizdo, V., Gilson, M.K.: Thermodynamic and differential entropy under a change of variables. Entropy 12, 578-590 (2010). https://doi.org/10.3390/e12030578

36. Li, Z., Li, W., Liu, R.: Applications of entropy principles in power systems: A survey. In: IEEE/PES Transmission and Distribution Conference \& Exhibition: Asia and Pacific, Dalian, China, pp. 1-4 (2005).

37. Tsallis, C.: Thermostatistically approaching living systems: Boltzmann-Gibbs or nonextensive statistical mechanics. Phys. Life Rev. 3(1), 1-22 (2006)

38. Castro, E., Moreno-García, P., Gallego, A.: Damage detection in CFRP plates using spectral entropy. Shock Vib. ID 693593 (2014)

39. González de la Rosa, J.J., Agüera Pérez, A., Palomares Salas, J.C., Sierra Fernández, J.M.: A novel measurement method for transient detection based in wavelets entropy and the spectral kurtosis: an application to vibrations and acoustic emission signals from termite activity. Measurement 68, 58-69 (2015) 
40. Piotrkowski, R., Castro, E., Gallego, A.: Wavelet power, entropy and bispectrum applied to AE signals for damage identification and evaluation of corroded galvanized steel. Mech. Syst. Signal Process. 23, 432-445 (2009)

41. El-Zonkoly, A.M., Desouki, H.: Wavelet entropy based algorithm for fault detection and classification in FACTS compensated transmission line. Electr. Power Energy Syst. 33, 1368-1374 (2011)

42. Jayasree, T., Devaraj, D., Sukanesh, R.: Classification of transients using wavelet based entropy and radial basis neural networks. Int. J. Comput. Electr. Eng. 1(5), 1793-8163 (2009)

43. Baxter, M.G., Pullin, R., Holford, K.M., Evans, S.L.: Delta T source location for acoustic emission. Mech. Syst. Signal Process. 21, 1512-1522 (2007)

44. Gallego, A., Martinez, E.: Emisión acústica. Niveles 1 y 2. AEND, Madrid (2015)

45. Rindorf, H.J.: Acoustic emission source location in theory and in practice. Bruel Kjaer Techn Rev 2, 3-44 (1981)

46. Gupta, A., Duke, J.C.: Identifying the arrival of extensional and flexural wave modes using wavelet decomposition of ultrasonic signals. Ultrasonics 82, 261-271 (2018)

47. Stepanova, L.N., Ramazanov, I.S., Kanifadin, K.V.: Estimation of time-of-arrival errors of acoustic-emission signals by the Threshold method. Russ. J. Nondestr. Test. 45(4), 273-279 (2009)

48. Andria, G., Attivissimo, F., Giaquinto, N.: Digital signal processing techniques for accurate ultrasonic sensor measurement. Measurement 30, 105-114 (2001)
49. Lokajicek, T., Klıma, K.: A first arrival identification system of acoustic emission signals by means of a high-order statistics approach. Meas. Sci. Technol. 17, 2461-2466 (2006)

50. Physical Acoustics Corporations: In: DiSP with AEWin user's manual. Princeton Junction, Princeton (2004)

51. Grosse, C.U., Ohtsu, M.: Acoustic emission testing. Springer, Berlin (2008)

52. Mártinez-Jequier, J., Gallego, A., Suárez, E., Juanes, F.J., Valea, A.: Real-time damage mechanisms assessment in CFRP samples via acoustic emission Lamb wave modal analysis. Compos Part B 68, 317-326 (2015)

53. Theobald, P., Zeqiri, B., Avison, J.: Couplants and their influence on AE sensor sensitivity. J. Acoust. Emiss. 26, 91-97 (2008)

54. ASTM: Standard guide for determining the reproducibility of acoustic emission sensor response, American Society for Testing and Materials, Vol. 976 (1994)

55. Hsu, N.N., Brecknbridge, F.R.: Characterization and calibration of acoustic emission sensors. Mater. Eval. 39, 60-68 (1979)

56. Nielsen, A.: Acoustic Emission Source Based on Pencil Lead Breaking, Vol. 15. The Danish Welding Institute Publication, 80, $1-15(1980)$

57. Suárez, E., Medida de ondas de Lamb en placas de material compuesto de fibra de carbono Master Thesis Project, University of Granada, Spain. 